\title{
Pragmatic Questioning's Core Linguistic Properties
}

\author{
Weihong Chen \\ College of Foreign Languages, Quanzhou Normal University, \\ Quanzhou 362000, Fujian, P. R. China \\ chwh518518@163.com
}

\begin{abstract}
Key Words: Linguistic Adaptation Theory, Variability, Negotiability, Adaptability.
\end{abstract}
\begin{abstract}
From the perspective of Linguistic Adaptation Theory, questioning is a special language use and speech act which consists of three core linguistic properties. To further explore its pragmatic qualities, the study implements a comprehensive study in questioning's variability, negotiability and adaptability based on data from TV interviews.
\end{abstract}

\section{Introduction}

During the past twenty years, together with questions, questioning has been studied from different approaches, such as sociolinguistics, psychological and pragmatic analysis and so on. The sociolinguistic approach focuses the relationship between questioning and social factors, while the psychological approach sheds light on psychological needs and intention in questioning. As for the pragmatic approach, it is particularly concerned with its functions as a kind of language use.

Thinking "questioning" as a dynamic activity, related researches regard it from the perspective of pragmatics. He Gang (1995) treated "questioning" as a speech act and process, which is driven and motivated by pragmatic needs and designed to realize certain communicative goals. The speaker utters the "questioning" to make it perform different communicative tasks in specific context. Therefore, "questioning" can be defined as the pragmatic need and verbal process of the speaker. In 1997, He Gang made further study and improved his observation on "questioning" by viewing it as an act full of various complicated features and interpersonal-situational interactive values. To make it easy to understand, he went on with explaining it in the ensuing part and found that "questioning" is an act with functional selectivity of the context and that serving the interactive purposes through the use of language. Here, in his study, he emphasized the importance and influence of context on "questioning" in illustrating the latter's pragmatic power and functions.

Seen from this, it can be concluded that from the pragmatic point of view, "questioning" is an important and universal behavioral mode to effectuate linguistic interpersonal functions in speech activities. Other pragmatic researches on it show that "questioning" can not only attract the guest's attention, transfer feelings, provide the guest's thought with fixed direction for eliciting more information, but also hold the tempo under control with the topic at the core and so on. Therefore, it is not exaggerated or overstated to say that "questioning" is a critical point to the success of conversation, which plays a vital and dominant role to promote conversations, especially in the TV interview programs featured typical "question-answer" pattern. Manipulation of the skill of "questioning" makes great contributions to a successful interview, which has become the host's top priority in preparation for interviews.

To further examine its pragmatic quality, based on Verschueren's Linguistic Adaptation Theory, the author will conduct a pragmatic analysis of questioning's core properties in TV interview programs selected from several episodes of three famous TV interview programs in China( Duihua, Yang Lan One on One and Lu Yи You Yue). 


\section{Three Core Properties of Questioning in TV Interview Programs}

To well understand the process of making choices, it is necessary to define some properties of language. Verschueren (1999) believes that there are three basic properties of language, which are variability, negotiability and adaptability. As a special kind of language use, questioning is also characteristic of these three features. According to his theory, questioning is constantly changing process, the utterance of which are made from a various and negotiable range of linguistic choices based on continuous adaptations to the changing interview contexts.

2.1 Variability of Questioning in TV Interview Programs-Basis of Negotiation and Adaptation. Variability of questioning means that there is a variability of possibilities from which language choices can be selected to produce a questioning utterance. Verschueren (1999) believes that the pragmatic perspective on language use or verbal action constructed covers a wider range than previous traditional pragmatic theories that defined variability as "varieties of language" geographically, socially, or functionally. Verschueren's argument indicates that variability contains any other possible options except what traditional theories defined. That is, variability may contain all structural levels of linguistic forms such as phonological and/or phonetic options, words and grammatical forms, intonation patterns to the extra linguistic level like social conventions, psychological motivations, culture, etc., which covers the entire range of variable options that must be assumed to be accessible to langue users for them to be able to make choices. Besides, it is dynamic and full of uncertainty of language use, which is synchronically and diachronically changing.

In the following part, the variability of questioning in TV interview programs will be discussed.

2.1.1The Structural Level. In TV interview programs, the variability of the host's questioning is mainly based on the syntactical and grammatical level. It is obvious that when uttering a questioning, there are tremendous ways and choices to select from. From the perspective of syntax, the host can utter his /her questioning in different forms: yes/no questions, wh-questions, alternative questions and tag questions, while from the perspective of discourse, he/she can articulate his/her questioning with presupposition, the third party tone, etc.

For example, to convey the same message, the host in a TV interview program has access to various syntactic forms.

Example 1

主持人: 实在跟我们想像当中太不一样了, 但是大前先生 11 年前, 凭什么您做出了这样 的一个判断? (It's really far from what we imagined. But Mr. Daqian, on what basis did you arrive at such a conclusion?)

Example 2

主持人: 这个听起来还是挺艰难的。刘教授, 您干嘛制造那么多障碍, 不见人家, 还得花 半年的时间? (This sounds very difficult. Professor Liu, why didn't you want to meet him until half a year later?)

\section{( 《对话》世界是平的 中国样本)}

The above two pieces of the host's questioning are all concerned about articulating his puzzle and/or disagreement to the guests, which greatly achieve successful communicative goals as the host wishes. In Example 1 and 2, the host chooses wh-questions with pre-sequences to utter his doubt and seek more information. To approach the same communicative goal, the host can choose any other ways of questioning. For example, to request for information, the host can ask the questions directly without any presupposition or pre-sequences. In terms of syntactical forms, except the above syntactical forms, there are still tremendous forms to select that can convey the same message to the guest. The first reason that the host chooses the above forms and ways of questioning is bases on his concern for the guest's face, who intends to reduce the verbal force of disagreement as possible as he could. The above questions are designed to challenge the guests' decision and behavior, to ask why they made such decision. If the host chooses direct forms of questioning without any presupposition, they will sound impolite and blunt, which may upset or even annoy the guest. The second reason that 
the host selects the above syntactical forms of questioning is that they can hide his true opinions and/or criticism, since neutralism is one of the primary principles that a host should abide by in interviews. Uttering in an interrogative form, the host can indicate his viewpoint as well as avoid criticism for his self-assertion by public.

Example3

杨澜: 那也是您第一次能够到桂林给自己的母亲章亚若女士扫墓。在您弟弟病危的情形下 到大陆, 那样的一种心情, 在您扫墓的时候是不是特别明显? ( It's the first time you were allowed to go to Guilin to visit your mother's grave. And then, your younger brother was critically ill. Therefore, did you feel very much more gloomy during that visit?)

\section{(《杨澜访谈》 章孝严)}

As is known, “您” is a counterpart of “你” in Chinese, both of which refer to the same person in conversation. The reason why the host chooses the linguistic choice of “您” instead of “你” in the interview is that the former is more often used in formal occasion to show respect for the hearers. The interview settings always embody some sorts of restrictions. Endowed with the right to present and manage the interaction in interviews, the hosts should be usually aware of the setting where he/she shapes his/her questioning, so as to make sure that his/her communication with the guest will not be impeded by improper forms of questioning as well as secure the success of the interview.

2.1.2 Social and Psychological Linguistic Factors. Any options that contribute to language use and can be selected by language users can be included while discussing the range of variability. The questioning used in TV interview programs makes no exception, the variability of which, except taking the linguistic forms mentioned above into consideration, also pays attention to other elements like social conventions, psychological motivations, etc. Some institutional speech events require fixed phrasings. Some settings or institutions have certain uniform expectations which define the coordinate interactions of individual within social systems and the appropriate behavior in social contexts (Shen 2004). That is, utterances of communicators in some settings should be sometimes restricted by the settings. As a presenter and moderator of an interview, the host should be aware of the interview setting where he/she utters his/her questioning. For example:

Example 4

杨澜: 您觉得台商现在的普遍意愿, 和目前台湾政治体现出来的主导力量有什么不同？(In your opinion, what is the difference between Taiwan businessmen's prevailing expectation and that of the dominant political party in Taiwan?)

\section{(《杨澜访谈》 章孝严)}

In the above conversation, the interviewer raises a sensitive topic to the guest-what is the difference between the popular Taiwan businessmen's opinion and the dominant political attitude towards the relationship between the mainland and Taiwan. In such an interview setting, the host is aware that the conversation is broadcast to the audience, which means that she is obliged to choose political phrasing to utter her questioning.

Extra linguistic factors also include psychological motivations and intentions. For example:

Example 5

杨澜：其实人性就是由各个方面的, 看你要去迎合哪一个方面? (I suppose humanity consists of multiple sides. The main question is that which side you prefer, right?)

张小燕：去迎合哪一方面？对，对。( Prefer a side? Yes, yes.)

\section{(《杨澜访谈》张小燕)}

Prior the host's questioning in Example 5, the guest described her attitude about revealing interviewee's privacy in entertainment programs, who doesn't think that can work forever in entertainment programs. After her statement, the host utters this questioning. In fact, this is the host's personal feel on humanity, which is designed to ask for the guest's agreement, for from the guest's statement in the previous turn, she supposes that the guest will agree with her. However, the guest misunderstands it at first, who repeats the host's questioning as to show her doubt and puzzle, 
although then she answers with "yes, yes". The variety of questioning in TV interview programs is large and profound, based on which the host can select any other ways to utter the above questioning. However, here the host chooses the above way, which is mainly driven by her intention, serves as a conclusion as well as an end of the stretch of talk.

Meanwhile, it should be pointed out that anything in the interview setting that can exert influences on the choice-making can be counted in the range of variability, such as bodily gesture, physical appearances, smiles, gazes, etc.

From the above analysis, we can conclude that questioning is characteristic of variability. That is, as a speech act, questioning in TV interview programs is faced with changing various linguistic choices.

\subsection{Negotiability of Questioning in TV Interview Programs-Principles of Negotiation.} Verschueren (1999:55) defines negotiability as a property accounting for the highly flexible principles and strategies that linguistic choice-making is based on instead of mechanically selection in accordance with strict set rules or fixed form-function relationship. Variability is the premise of making choices, while negotiability denotes the strategies and methods used in making choices. Both play a solid foundation for adaptability. Besides, negotiability concerns choice-making both of the language producer and the interpreter. In questioning in TV interview programs, it covers both sides of the speaker and the hearer. That means, abiding by related flexible principles, strategies and conventions, the host is faced with a wide range of alternatives. Meanwhile, the hearer (the guest being questioned, the audience at present or the viewers in front of TV sets) is also encountering tremendous choices to interpret the same questioning the host just uttered.

2.2.1 Cooperative Principles. When uttering his/her questioning, the host will negotiate with himself to select a choice from a wide range of linguistic choices of questioning. Basically, he/she will be compliance with Grice's Cooperative Principles into consideration, which contains four maxims: quantity maxim (make your contribution as informative as is required, but not more, or less, than is required); quality maxim(do not say that which you believe to be false or for which you lace evidence); relation maxim ( be relevant) and manner maxim ( be clear, brief and orderly). A successful conversation should be the fruit of all the participants' efforts and collaboration is a necessary factor for them to achieve certain goals. Therefore, people are expected to cooperate during a conversation. The host, who is responsible for the smooth development of conversation, will then make sure that her questioning will not cause any obstacles to the further interaction.

Example 6

杨澜: 你是不是一直有意识地使自己和这个圈子保持一定的距离？(Question 1)

(Have you kept a distance from the circle of entertainment intentionally?)

陈道明: 我不是有意识的。我在圈子里有几个谈得来的朋友，与其他人交往话不多。上酒 桌对我来说是一种煎謷。(No. I didn't do it on purpose. I do have a few genuine friends in the circle. However, I seldom hang out with others. Besides, wine-drinking culture in China is a torture to me.)

杨澜：没话说。（Having nothing to say.）

陈道明: 没话说......(Yes, I find nothing to communicate with them.)

杨澜：你觉得好像是别人强加给你的？(Question 2)

(Do you feel compelled to communicate with some guys sometimes?)

陈道明：对。我还得客客气气地跟他说: “好, 听着呢。”(Yes. And I have to pretend that I am listening .)

杨澜：那你跟什么样的人在一起话越来越多呢？真要是跟钱钟书坐在一块儿聊天你的话 多吗? (Question 3)(Then, which type of people you prefer to speak up? With Qian Zhongshu?)

陈道明：没，更没话说了。(Er, not with him. Then we have less to share.)

(《杨澜访谈录》: 陈道明)

In the above example, the host's three pieces of questioning is well in line with three maxims of cooperative principles: quality, relation and manner. In terms of quality, the host appropriately utters 
her questioning connected with the second part of the previous adjacent pair, expecting the guest to continue to confirm it or explain it. The maxim of quality turns out to be a general principle of speech act theory and holds for all types of utterances. For example, here, we can find that the host tends to exploit the quality maxim by relying on facts that the guest has related in her uttering Question 2 . And all questioning in the above is related, which are all concerned about the guest's social contact in the circle of entertainment and which can be therefore seen as compliance with the relation maxim. All of her questioning utterances are relevant to the ongoing conversation. And obviously, the above underlined pieces of questioning are all articulated in a clear and orderly way, all of which, though simple and short, clearly expresses her motivations, and all of which are accurately interpreted by the guest. The host's cooperative questioning, therefore, creates a friendly communicative atmosphere, which greatly wins the guest's positive response who always keeps a low profile and seldom speaks in public. So, it is obvious while the host negotiates with herself on what linguistic choices to shape her questioning, she may take cooperative principles into consideration, which, thus, leads to the above questioning utterances.

2.2.2 Politeness Principles. Linguistic politeness has been penetrated in great detail by various linguistic from a variety of perspective. Brown and Levinson $(1978,1987)$ 's face-saving view plays an important part in the research of politeness. The concept of "face" acts as the core of the two linguists' theories on politeness, which refers to the individual's public self-image. According to participants' wants, face can be divided. The former is defined by Brown and Levinson (1987) as " the want of every 'competent adult member' that his action be impeded by others", the "want to have his freedom of action unhindered and his attention unimpeded" and the latter as " the want of every member that his wants be desirable to at least some others" and the "perennial desire that his wants (or the actions/acquisitions/values resulting from them should be thought of as desirable)". The premise of the view is that it benefits every participant to maintain one another's face and to reveal this intention to others during communication. To soften the intrinsically face-threatening acts (FTAs), one turns to a couple of possible strategies or goes off record. The redresive strategies consist of positive politeness and negative politeness strategies. Brown and Levinson propose lists of linguistic means to realize the politeness strategies. A number of the linguistic means, when employed in a given interaction, rely on interrogatives as their linguistic forms. Many of the questioning from the data function as the realization of the politeness strategies.

In TV interviews, the host always concerns the guest's feeling, emotion, and opinion while questioning, as to minimize the verbal negative force his/her questioning might create. His/her negotiability on linguistic choices of questioning is always in compliance with several politeness strategies like mitigating disagreement, seeking agreement, presupposing/raising/asserting common ground, compliment, sympathy arousing, etc.

Example 7

鲁豫: 刚才我们看到男演员把身探过去, 要亲诺拉·琼斯, 听说这个拍了一百遍? ( I learned that this scene is shot for a hundred times?)

王家卫: 对, 一百多条。(Yes, more than one hundred.)

鲁豫：要拍那么多条吗? 你最后用了多长? (Is it necessary? How many you adopted finally?) 王家卫: ...... (Answers are omitted here)

\section{(《鲁豫有约》: 王家卫: 另类映像)}

In the above example, the underlined questioning, in fact, indicates the host's disagreement who doesn't feel that it is necessary to repeat for so many times. But, to show her respect for the guest and evade blunt offence to the guest, the host avoids showing her opinion directly by saying "I don't think it is necessary to repeat for so many times". The host's questioning is based on her negotiation on mitigation strategy so as to avoid threatening the guest's face.

Therefore, based on the above analysis, it is apparent that questioning is a locutionary act, which is possessed with the property of variability and negotiability. That is, questioning is a dynamic process 
made up the continuous linguistic choice-making from various linguistic choices as well as driven and motivated by highly flexible principles and strategies.

And then, here comes to the question: if questioning is a process of constant negotiation and renegotiation, how can communication be successful? The possible success lies in the third feature of language - adaptability. According to Linguistic Adaptation Theory, adaptability mainly decides the choice-making and the final utterance. Therefore, variability, negotiability and adaptability are fundamentally inseparable, which are interrelated notions of questioning and the hierarchical ranking of which is a conceptual tool to come to grips with the complex pragmatic linguistic phenomenon-questioning. Among these three notions, adaptability is the higher level of the hierarchy, which contains no content without both variability and negotiability. And the following part will carry out a detailed and concrete exploration into the adaptability of questioning in TV interview programs.

2.3 Adaptability of Questioning in TV Interview Programs-Results of Continuous Negotiation. Adaptability (Verschueren, 1999:61)is the property of language which enables human beings to make negotiable linguistic choices from a variable range of possibilities in such a way as to approach points of satisfaction for communicative needs.

In TV interview programs, the host is a special speech act, which can fulfill many pragmatic functions in changing specific interview contexts. The host utters his/her questioning, which is then interpreted and answered by the interviewees. Besides, in order to seek information from the host, the host always makes his/her questioning utterances based on negotiating with various options at different structural levels and contexts. First, he/she will pay attention to the interview setting and chooses proper language. Secondly, in accordance with social conventions, he/she will make his/her questioning sound as polite as he/she could. Thirdly, to realize various communicative goals, he/she will consciously choose appropriate questioning based on his/her constantly changing psychological world. Therefore, the host's questioning is a dynamic and interactional process of adaptation, which indeed is the actual concrete realization of adaptation to such three contexts: physical environment, social conventions and psychological motivations.

2.3.1Questioning in Interview Programs as Adaptation to the Interview Environment. Environment contains all objective existing material elements that exert influences on linguistic choices of communication is defined as the physical world, in which time and space are the most common components, for these two are the most obvious concepts in our real life in physical world. Therefore, they have received much concern and have been many linguists' top priority of research. Time is an essential issue that none interview program will neglect or ignore it. The time when an interview program is presented or broadcasted to the audience directly influences its audience rating. In order to attract as many audiences as possible, interview programs are always shown at the prime time every night or during the weekends so as to adapt to the viewers' viewing habits. Although interview programs need not to be broadcasted just after an event happens just as news reports do, when choosing topics of the interview program, the importance of the time factor allows of no neglect. On the whole, events happen just recently both at home and abroad are always priority choices on the topics of interview programs, especially those which are arousing great and worldwide attention in society. For example, with the forthcoming $60^{\text {th }}$ anniversary of the founding of the People's Republic of China, many interview programs tend to choose topics related to it to adapt to the audience's interest and concern. And before the presidential election, American TV studios make a lot of interview programs on the president election in favor of different popular candidates, so as to adapt to audience's great attention on this issue. Therefore, the time of event does have an enormous effect on the interviewers' choices and content of questioning.

Besides, if the concept of time appears in the hosts' questioning, its concrete meaning should be identified and understood based on specific contexts. For example:

Example 8 
主持人: 从来的那一刻到现在, 你们的感触是什么? (How do you feel from the moment you arrived at our studio till now?)

\section{(《对话》 世界是平的 中国样本)}

According to Jef Verschueren, there are three types of time: event time, time of utterance and reference time. In the above example, we can see that the host based his questioning on the time when the interview was recorded. Here, the time "from the moment you arrived at our studio till now" denotes these time phrases' literal meaning. Thus, here, the host takes the perspective of event time, which is also the adaptation to time of receipt. Since the host and the interviewer are communicating with each other here in the studio face to face, it is common to ask how they feel during the process of the communication, which will make the communication continue more smoothly and harmoniously. Moreover, when the host asks the question, "the moment you arrived at our studio till now" can greatly draw the audience's attention at present, for they are there watching the whole process of the interview, and they are concerned about the interviewee's real thought about the whole communication and the topic they are discussing.

2.3.2 Questioning in Interview Programs as Adaptation to Social Conventions. The social world includes norms of a society that have come into being gradually in daily life and can be reflected in clothing, food, shelter and transportation, etc. It is changeable, regional, in which culture is embedded. Language use does serve as reflection to society and culture. As a specific linguistic phenomenon, questioning can also act as carrier of some social norms and cultural elements. Meanwhile, social norms and cultural elements will also play a role in the shaping and molding of questioning in TV interview programs.

\section{Example 9}

主持人: 跟我们来分析一下, 比如说在中国的媒体和海外的媒体, 尤其是法国的媒体, 对你的这番评价当中最让你认可的, 你觉得真的是起到了自己的这种作用的评价会是什么样 的评价? (Now, please analyze with us, from so many comments made by the media from home and abroad, especially the French media, which do you think really works to you?)

李洹: 我觉得最重要的两个词应该是真诚和理性。

(I suppose that these two words—-sincerity and ration—are the most important ones.)

(《对话》: 火炬传递什么)

In example 9, the host chose the first plural pronoun “我们 (us )" before he uttered the questioning. Obviously, here, it is only he who really participates in the analysis with the guest to judge which is the most suitable comment the guest supposes to himself. But the host chose to use "us" instead of the word "me", so as to make an adaptation to show his respect for every participant in the interview setting. It is polite to take everyone at present into consideration, to make each of them feel that they are not neglected. Therefore, on hearing such similar questioning with the first plural pronoun, viewers (especially the audience at the studio) will feel being concerned and respected by the host. Such can cultivate a whole amicable air for the interview, making everyone at present feel comfortable and friendly.

Meanwhile, the host will pay much more attention to the guest's social status. Social status is understood as the degree of honor or prestige attached to one's social position. It is a hierarchy of perceived and often accepted social superiority. Social status is a key component in China's culture and society. The Chinese pay much attention to social status, for which they will show and keep not only through dress, manners and demeanor but also through language. It is regarded as one of the basic ways to judge whether a man respects others by judging whether his language well and accurately reflects the social status of the listener. In interview programs, when using questioning, in order to show his/her respect to the guest, the host will choose language that can accurately show the social status of the guest.

2.3.3 Questioning in Interview Programs as Adaptation to the Interviewer's Motivations. Verbal interaction is no doubt communication from mind to mind (Verschueren 1999:87). Linguistic choices are affected by communicators' "minds in society" more or less. The mental world activated 
in language use contains emotive and cognitive elements. In the present study, it intends to check whether the host's motivations and intentions will play a critical role in constructing his/her questioning discourse during the interview process.

Example 10

主持人: 其实我想对于北川中学的老师来说, 他们的压力会非常大, 并不仅仅来自于工作上 的压力, 可能自己情感上、心理上的这些压力, 同样每时每刻都和他们相伴, 是这样吗

(Indeed, I think that teachers in Beichuan Middle School are under great pressure, which is not only caused by their job. You are also under emotional pressure continually, right?)

寒绍琪: 是, 有这个事实是, 但是我说过的, 地震再强, 它震不垮我们北川中学师生的这个 意志和精神, 我们一定会重新站起来。(Yes. It is true. But just as I said once before, the earthquake could not quake our willingness, strength and spirits no matter how mighty it seems. We will be strong again.)

\section{(《对话》: 当灾难降临)}

Questioning as eliciting appreciation and approval is always used in discussing serious social issues that the public are extremely concerned with the guest. In the above example, the host talked of the enormous pressure that teachers in Beichuan Middle School have been bearing, who have just survived the earthquake in May, 2008. He mentioned their pressure by questioning, which is not designed to inquire information but to seek the guest's agreement for his statement on their emotions and pressure and which can therefore seek the common psychological ground between the host and the guests. And then, the questioning can greatly incur the audience's concern for the guest, and some will even feel very sorry for their situations. Therefore, the host's questioning here as an adaptation to seek the guest's approval and appreciation.

\section{Summary}

Based on Verschueren's Linguistic Adaptation Theory and data collected from three famous China's interview programs, the study carries out a full study of questioning's three core linguistic properties, which serve as the logic foundation for the dynamic generating of questioning utterances. From the above detailed analysis, the author come to the conclusion that variability, negotiability and adaptability are three inseparable components of questioning as a special speech act, which then play an important role in questioning utterances generating and its adaptation to contexts.

\section{Acknowledgements}

This work was financially supported by Social Science Research Pragramme of Fujian (FJ2016C157) and pre-research Foundation of National Social Science Foundation of Quanzhou Normal University(2016YYS K11).

\section{References}

[1] He Gang. On Cultural Functions of Pragmatic Questioning [J]. Journal of Shanghai International Studies University,(1995); (In Chinese).

[2] Verschueren, J. Understanding Pragmatics[M].Beijing: Foreign Languag Teaching and Research Press, 1999.

[3] Brown P., \& S. Levison. Universals in Language Usage: Politeness phenomena[A].In Goody N. (ed.), Questions and politeness[A].Cambridge: Cambridge University Press, 1978. 